\title{
EQUILIBRIUM OF A PRESTRESSED STRIP REINFORCED WITH ELASTIC PLATES
}

\author{
N. N. Dikhtyaruk
}

UDC 539.3

The contact problem for a prestressed elastic strip reinforced with equally spaced elastic plates is considered. The Fourier integral transform is used to construct an influence function of a unit concentrated force acting on the infinite elastic strip with one edge constrained. The transmission of forces from the thin elastic plates to the prestressed strip is analyzed. On the assumption that the beam bending model and the uniaxial stress model are valid for an elastic plate subjected to both vertical and horizontal forces, the problem is mathematically formulated as a system of integro-differential equations for unknown contact stresses. This system is reduced to an infinite system of algebraic equations solved by the reduction method. The effect of the initial stresses on the distribution of contact forces in the strip under tension and compression is studied.

Keywords: contact problem, prestressed elastic strip, elastic plates, unit concentrated force, integro-differential equations, Fourier integral transform, reduction method, contact stresses

1. Introduction. Formulation of the Problem. Boundary Conditions. The distribution of contact stresses between periodically placed elastic plates and an elastic isotropic half-plane and a strip in the classical theory of elasticity was studied in [9]. Contact problems for a half-plane and half-strip in the linearized theory of elasticity were analyzed in [2-4, 6, 8, 10-12].

In the present paper, we will analyze, within the framework of the linearized theory of elasticity [1, 2], the influence of the initial (residual) stresses on the distribution of contact stresses in a prestressed elastic strip reinforced with elastic plates of length $2 a$ spaced at $2 l(l>a)$.

We will describe below a method for solving the problem posed. Assume that the initial state of the nonreinforced elastic strip is homogeneous and $S_{22}^{0}=0$, i.e., the strip is loaded only along the $O y_{1}$-axis. We will use the coordinates $y_{i}$ of the initial stress state, which are related to the Lagrange coordinates (of the natural state) by $y_{i}=\lambda_{i} x_{i}, i=1,2$, where $\lambda_{i}$ are the coefficients of expansion determining displacements of the initial stress state.

We will perform our analysis in the general form using the theory of large (finite) initial strains and arbitrary elastic potentials.

Assume that thin elastic plates are bonded onto a prestressed elastic strip along finite sections $L_{k}\{-a+2 l k ; a+2 l k\}$, $k=1,2, \ldots, n$. Concentrated external forces $P$ are applied to one of their ends. The contact stresses arising in the plate and strip on the contact areas $L_{k}$ are $2 l$-periodic functions $[2,10]$, i.e.,

$$
\begin{gathered}
\tau_{x y}^{(1)}\left(y_{1}\right)=\tau^{(1)}\left(y_{1}\right)=\tau^{(1)}\left(y_{1}-2 l\right)=\tau^{(1)}\left(y_{1}+2 l\right), \\
\widetilde{Q}_{21}\left(y_{1}\right)=T\left(y_{1}\right)=T\left(y_{1}-2 l\right)=T\left(y_{1}+2 l\right) .
\end{gathered}
$$

Moreover, the following contact conditions must be satisfied on $L_{k}[2,10]$ :

$$
u\left(y_{1}\right)=u_{1}\left(y_{1}\right), \quad v\left(y_{1}\right)=u_{1}\left(y_{1}\right), \quad \forall\left(y_{1}\right) \in L_{k}, \quad y_{2}=0
$$

Podol'e University of Technology, Khmel'nitskii, Ukraine. Translated from Prikladnaya Mekhanika, Vol. 40, No. 3, pp. 63-70, March 2004. Original article submitted February 7, 2003. 
where $u$ and $v$ are the horizontal and vertical displacements of the elastic plate and $u_{1}$ and $u_{2}$ are the displacements of the elastic strip.

Since the elastic plates are arranged periodically, the effect of the initial stresses on the contact areas $L_{k}$ at $y_{2}=0$ will be identical $[2,9]$. Therefore, it is enough to consider just one elastic plate $L_{0}(k=0)$. In addition to (1.2), the ordinary static equilibrium conditions for the elastic plate must be satisfied $[2,9]$.

Let us analyze the equilibrium conditions for an elastic plate of finite flexural stiffness. Its elastic modulus $E_{1}$ is much greater than the elastic modulus $E_{2}$ of the prestressed strip, which is due to the smallness of the thickness $h$ of the elastic plates. Then the bending and uniaxial strain equations can be written as $[2,9]$

$$
\begin{gathered}
D \frac{d^{4} v}{d y_{1}^{4}}=p\left(y_{1}\right)-p_{0}\left(y_{1}\right), \quad\left|y_{1}\right| \leq a, \\
\frac{d v}{d y_{1}}=\frac{1}{E_{1} h} \int_{-a}^{y_{1}}\left[q(\tau)-q_{0}(\tau)\right] d \tau, \quad\left|y_{1}\right| \leq a,
\end{gathered}
$$

and the above-mentioned static equilibrium conditions must be met at the contact area $L_{0}\{-a ; a\}$. Here $p\left(y_{1}\right)$ and $q\left(y_{1}\right)$ are the normal and tangential contact stresses, and $p_{0}\left(y_{1}\right)$ and $q_{0}\left(y_{1}\right)$ are the horizontal and vertical forces applied to the elastic plate.

Thus, the task is to analyze the effect of the initial (residual) stresses in the elastic strip on the distribution of the normal $p\left(y_{1}\right)$ and tangential $q\left(y_{1}\right)$ contact stresses within the contact area $L_{0}\{-a ; a\}$. Note that the solution to be discussed below is valid only when the elastic strip is under plane strain; and the uniaxial stress-strain model is assumed valid for the elastic plates $[2,9]$. We will use the notation adopted in the theory of elasticity [9] for the quantities pertaining to the elastic plates, and the notation from $[1,2]$ for the quantities associated with the elastic strip.

2. Governing Functional Equations. We will perform our analysis in the general form, adhering to the linearized theory of elasticity for compressible and incompressible bodies with arbitrary elastic potentials. For the theory of large (finite) initial strains, it is necessary to introduce the simplifications mentioned in [1].

To solve the problem posed, we will use the solutions for compressible and incompressible bodies in the coordinates of the initial strain state $y_{i}$. It is assumed that the initial state of the strip is homogeneous and $[1,2]$

$$
u_{m}^{0}=\left(\lambda_{m}-1\right) x_{m}, \quad \lambda_{m}=\text { const }
$$

where $u_{m}^{0}$ is the displacement that determines the initial state of the strip with homogeneous initial stresses.

The stresses and displacements of the strip boundary in the case of equal and unequal roots of the constitutive equation [2] are defined by

$$
\begin{gathered}
u_{1}\left(y_{1}, z_{i}\right)=\frac{i}{2 \pi} \int_{-\infty}^{\infty}\left[L^{+}\left(A_{1}+A_{2}\right)+\alpha z_{1} L^{-}\left(B_{1}+B_{2}\right)\right] \exp \left(-i \alpha y_{1}\right) d \alpha \\
u_{2}\left(y_{1}, z_{i}\right)=\frac{m_{1}}{2 \pi \sqrt{n_{1}}} \int_{-\infty}^{\infty}\left[L^{+}\left(B_{1}+s_{1} B_{2}\right)+\alpha z_{1} L^{-}\left(A_{1}+s_{1} A_{2}\right)\right] \exp \left(-i \alpha y_{1}\right) d \alpha \\
\widetilde{Q}_{22}\left(y_{1}, z_{i}\right)=\frac{C_{44}\left(1+m_{1}\right)}{2 \pi} \int_{1}^{\infty} \alpha\left[L^{+}\left(A_{1}+s A_{2}\right)+\alpha z_{1} L^{-}\left(B_{1}+s B_{2}\right)\right] \exp \left(-i \alpha y_{1}\right) d \alpha \\
\widetilde{Q}_{21}\left(y_{1}, z_{i}\right)=\frac{i}{2 \pi} \cdot \frac{C_{44}\left(1+m_{1}\right)}{\sqrt{n_{1}}} \int_{-\infty}^{\infty}|\alpha|\left[L^{+}\left(B_{1}+s_{0} B_{2}\right)+\alpha z_{1} L^{-}\left(A_{1}+s_{0} A_{2}\right)\right] \exp \left(-i \alpha y_{1}\right) d \alpha
\end{gathered}
$$

where $z_{j}=\left(n_{j}\right)^{-1 / 2} y_{2}(j=1,2), s_{0}=\frac{1+m_{1}}{1+m_{2}}, s=s_{0} \frac{l_{2}}{l_{1}}, s_{1}=\frac{m_{2}-1}{m_{1}}$, 